OAI-PMH: http://www.indteca.com/ojs/index.php/Revista Scientific/oai

Artículo Original / Original Article

\title{
Competencias Tutoriales en los Programas de Postgrado: Una Mirada desde la Experiencia Venezolana
}

Autoras: Carmen Consuelo López De Solórzano Universidad Pedagógica Experimental Libertador, UPEL clopez@impm.upel.edu.ve

Barinas, Venezuela Mariela Sofía Pérez Rodríguez Universidad Pedagógica Experimental Libertador, UPEL marielasofi@gmail.com

Lara, Venezuela

\section{Resumen}

La Tutoría representa un componente fundamental en los Programas de Postgrado; su acción trasciende lo meramente académico y consolida la formación en investigación de sus participantes, traduciéndose en calidad de producción intelectual y progreso social. El propósito de la investigación de tipo documental es proporcionar aportes teóricos referenciales, relacionados con los estudios de Postgrado en Venezuela, así como abordar la concepción, caracterización, tipología y operacionalización de la competencia tutorial. Este abordaje referencial forma parte de un estudio de mayor alcance denominado Competencias Tutoriales para la producción de textos académicos en el nivel de postgrado. Es así como, desde el ejercicio reflexivo, surge un conjunto de consideraciones sobre aspectos trascendentales de la competencia tutorial en la que prevalece la imprescindible formación en el plano investigativo. Desde esta visión se espera promover procesos reflexivos tendentes a generar mayor interés por programar acciones significativas en esta área la cual precisa de constante revisión sistemática y autorreflexión.

Palabras clave: tutoría; competencias del docente; postgrado; programas de educación. 


\section{Tutorial Competences in Postgraduate Programs: A Look from the Venezuelan Experience}

Abstract

The Mentoring represents a fundamental component in the Postgraduate Programs; its action transcends the merely academic and consolidates the research training of its participants, translating into the quality of intellectual production and social progress. The purpose of the documentary type research is to provide reference theoretical contributions, related to postgraduate studies in Venezuela, as well as to address the conception, characterization, typology and operationalization of the tutorial competence. This referential approach is part of a larger study called Tutorial Competences for the production of academic texts at the graduate level. This is how, from the reflective exercise, a set of considerations emerges about transcendental aspects of the tutorial competence in which the indispensable formation in the investigative plane prevails. From this perspective, it is expected to promote reflective processes aimed at generating greater interest in scheduling significant actions in this area, which requires constant systematic review and self-reflection.

Keywords: tutoring; teacher qualifications; postgraduate; education programs.

Date Received: 22-02-2018

Date Acceptance: 03-05-2018 


\section{Introducción}

La Tutoría representa una de las acciones protagonistas en el desarrollo de los Programas de Postgrado en Venezuela. Concebida no como un fin sino como un medio, constituye un recurso o dispositivo trascendental en el acompañamiento, orientación y guía de procesos de investigación propios de ese nivel. Como se describirá a lo largo del presente artículo, esta función es fundamental por cuanto permite garantizar la efectividad en el cumplimiento de los propósitos establecidos para este tipo de estudios universitarios. No obstante, la función tutorial se encuentra signada por carecer de una formación sistemática para su ejecución. Sobre esta problemática se refiere Ríos en el prólogo escrito a Ruiz (2006a):

...a pesar de lo importante y delicado del rol del tutor, su formación viene a ser una especie de "tierra de nadie" donde parece que cada cual "hace lo que puede" y va aprendiendo de su propia experiencia, con poca o ninguna instrucción sistemática...de allí la necesidad de una reflexión sobre la práctica... que permita ofrecerles a otros la posibilidad de mejorar su labor (pág. 16).

La cita precedente permite evidenciar la inquietud por parte de la comunidad universitaria en atender la problemática generada en torno al abordaje científico que existe en relación con las competencias tutoriales. La compleja tarea del tutor requiere reflexión, sistematización e instrumentalización, a fin de evitar una acción tutorial regida por las creencias y experiencias individuales sin la necesaria reflexión al respecto.

La dinámica de los Programas de Posgrado en Venezuela revela múltiples particularidades que atender, resolver y consolidar en atención a la productividad de cada ámbito del quehacer investigativo de dichos estudios universitarios. Con relación a esto, algunos estudios efectuados (Balbi, 2011a; Rondón, 2013; Terán, 2012a): coinciden en señalar como diagnóstico de la situación la baja productividad en la producción de trabajos de grado, 
encontrando entre los factores causales la necesidad de una orientación tutorial más efectiva. Si bien esta problemática obedece a múltiples factores, resulta inequívoca la relevancia que posee la acción tutorial en el éxito del proceso.

El presente artículo forma parte de una investigación mayor denominada "Competencias Tutoriales para la producción de textos académicos en el nivel de postgrado". Dicho estudio se ha venido desarrollando en los Programas de Postgrado acreditados para instituciones oficiales y privadas de educación universitaria pertenecientes a la región de los llanos occidentales venezolanos. En atención a ello, se propone compartir parte del camino referencial abordado, así como promover procesos reflexivos tendentes a generar mayor interés por programar acciones significativas en esta área la cual precisa de constante revisión sistemática y autorreflexión.

Es así como, desde el ejercicio reflexivo, surgen un conjunto de consideraciones sobre aspectos trascendentales en esta práctica. Específicamente, se espera proporcionar aportes teóricos referenciales relacionados con los estudios de Postgrado en Venezuela, destacando aspectos puntuales de su normativa que permiten darle sentido a la acción tutorial. Seguidamente se promueve el conocimiento de algunas concepciones de los términos Tutoría y Tutor, así como la historia, caracterización y tipología de dichos vocablos. Finalmente se aborda la operacionalización de las competencias tutoriales en el nivel de postgrado, no únicamente en el contexto educativo sino en el cumplimiento de la función de investigación; esto desde la consideración de las instituciones educativas universitarias como centros y espacios para la creación y desarrollo científico- social, hasta llegar a la colectividad y el individuo como agentes protagonistas de sus propios procesos de transformación, conversión y mejoramiento de esta acción.

Con el presente artículo se espera contribuir con un cuerpo firme de ideas y referentes dirigidas a vigorizar los procesos de acompañamiento 
tutorial, a través del desarrollo de competencias en esta área, especialmente en el nivel de postgrado.

\section{Contexto temático y Abordaje teórico}

\subsection{Programas de Postgrado en Venezuela}

En Venezuela, los estudios de postgrado corresponden al nivel más alto del sistema educativo perteneciente al subsistema de educación universitaria. En el mismo se desarrollan un conjunto de acciones y procesos técnicos, científicos, humanísticos, educativos y de producción intelectual, con miras a la ampliación y profundización del conocimiento en ámbitos de incumbencia particulares que requieren ser abordados en pro del desarrollo integral de la nación, así como las instituciones que la integran, enfocados en brindar aportes con relevancia social.

Las últimas dos décadas se han caracterizado por un incremento de éstos, así como la obtención de títulos en este nivel. Para Bello (2003): el crecimiento de los postgrados se puede medir (entre tantas variables) con base en el número de profesionales que se inscriben en este tipo de programas con el fin de obtener la certificación correspondiente; “...además este crecimiento, dado por producción-desarrollo y docencia-investigación es abruptamente alterado por un crecimiento matricular que se refleja al pasar de un aproximado de 6.000 cursantes para 1989, a unos 70.000 cursantes de postgrado para 1999" (pág. 70). Cabe destacar que hasta el año 2015 no solamente ha venido aumentando la matrícula, sino que proporcionalmente el número de programas de postgrado se ha visto incrementado.

El órgano encargado de analizar y evaluar las consultas para aprobar y acreditar los diferentes Programas de Postgrado en Venezuela es el Consejo Consultivo Nacional de Postgrado (CNU, 2001a, artículo 8). Dicho organismo técnico-asesor del Consejo Nacional de Universidades (CNU), se encarga de asignar las comisiones para revisar los estudios de factibilidad que en esta 
materia realizan las universidades. Hasta el 04 de marzo de 2018, dicho organismo señala en su portal oficial (CCNP), que en Venezuela existen mil ciento diez (1110) Programas de Postgrado autorizados. Específicamente, en las Universidades Oficiales se cuentan con 799 Programas autorizados, de los cuales trecientos ochenta y nueve (389) son Especializaciones, doscientos ochenta y dos (282) corresponden a Maestrías y ciento ocho (108) a Doctorados. De ese total, la Universidad Pedagógica Experimental Libertador (UPEL) cuenta con el mayor número de autorizaciones, para un total de ciento cuarenta y cuatro (144), de las cuales setenta y dos (72) son Especializaciones, cincuenta y siete (57) maestrías y quince (15) Doctorados. Es importante acotar que la autorización de un Programa de Postgrado constituye la aprobación, para su creación y funcionamiento, por parte del Consejo Nacional de Universidades (CNU) previa recomendación del Consejo Consultivo Nacional de Postgrado (CCNP, 2018).

En Venezuela también existe la figura de acreditación para los Programas de Postgrado; dicho proceso representa un acto voluntario de las Universidades en las que el CNU, luego de evaluar dicho Programa, reconoce públicamente que el mismo reúne los requisitos de ley establecidos en esta materia. Los Programas de Postgrado acreditados por CNU hasta la fecha son seiscientos ochenta y cinco (685), de los cuales uno (1) es de Especialización Técnica, trescientos seis (306) son Especializaciones, trescientos cuatro (304) Maestrías y setenta y cuatro (74) Doctorados. De este total la Universidad Central de Venezuela (UCV) es la institución oficial con mayor número de Programas de Postgrado acreditados, para un total de doscientos treinta y uno (231), de los cuales ciento veinte seis (126) son de Especialización, setenta y siete (77) de Maestría y veintiocho (28) Doctorados.

Así mismo, es importante acotar que en Venezuela, existe una normativa que regula los estudios en el referido nivel. La misma se denomina Normativa General de los Estudios de Postgrado para las Universidades e 
Instituciones debidamente autorizadas por el Consejo Nacional de Universidades. En la misma se establecen un conjunto de lineamientos que rigen las acciones y estructura organizativa encargada de atender esta materia; específicamente en lo relacionado con los Programas de Maestría, dicha normativa elaborada por el Consejo Nacional de Universidades (CNU, 2001b, artículo 24), plantea que: para la obtención del grado de Magister se exige la aprobación de veinticuatro (24) Unidades Crédito en actividades curriculares, así como la elaboración y aprobación de un Trabajo de Grado, asistido por un Tutor. Esto último representa un aspecto importante que atender para el contexto de lo planteado en el artículo, por cuanto dicho Trabajo debe ser elaborado por el estudiante con el apoyo de un Tutor.

\subsection{Tutor y Función Tutorial: historia, concepciones, tipología y reflexiones}

Antes de iniciar algunas consideraciones en relación con los tutores y sus competencias, es importante clarificar el umbral etimológico de la palabra tutoría con el fin de contrastar su origen con la aplicación actual del vocablo. A su vez, este recuento pudiera convertirse en un elemento para la reflexión y posible conversión del conocimiento en educación universitaria (López, 2016): por cuanto, para la consolidación de acciones que permitan asumir verdaderamente los aportes y tendencias generadas desde la esencia de cada función y proceso educativo a desarrollar, se requiere conocer el origen de los mismos con el fin de rescatar lo esencial, ajustando las estrategias.

Etimológicamente la palabra tutoría proviene del latín tutor, tutor-óris que significa protector y éste, al mismo tiempo, surge del verbo tuerique quiere decir observar o vigilar. Más en detalle destaca el hecho de que la palabra se conforma por la agrupación de tres núcleos que son: tueri (sinónimo de vigilar o proteger), tor que significa agente y el sufijo ia que equivale a cualidad. Desde esta perspectiva, el vocablo tutoría representa la autoridad para 
proteger o amparar a una persona.

Dicha función ha sufrido un conjunto de adaptaciones, especialmente a los fines que debía cumplir el tutor; éstos van desde pares que debían asistir a los más novatos, pasando por la ayuda gradual de docentes en asesorías y vigilancia a las conductas estudiantiles en el nivel de pregrado, hasta el cumplimiento de funciones educativas y didácticas de acompañamiento y mentoreo a estudiantes de postgrado para la realización de sus Trabajos de Grado. En este sentido, representa un gran aporte lo reseñado por Benedito, Ferrer y Ferreres (1995): quienes refieren como el sistema tutorial en la Gran Bretaña constituye una de las estrategias didácticas mayormente usadas constituyendo una práctica para la orientación del aprendizaje y el seguimiento intelectual del estudiante consistente en la realización de un trabajo escrito en el que desarrolle su capacidad crítica, el cual debe ser orientado (tutorado) por un experto, mientras que en las universidades anglosajonas se enfatiza en una educación más individual con profundidad antes que en la amplitud de conocimiento, lo que aquí se le denomina mentoría o monitoreo.

Conocidos estos dos enfoques macro, vale la pena precisar el sistema tutorial inglés, el cual según Ruiz (2006b), tiene las siguientes características:

(a) constituye una modalidad instruccional centrada en el estudiante; (b) la interacción académica está centrada en la discusión tutor-alumno; (c) el tutor y el estudiante se reúnen, por lo menos, una vez a la semana, durante una hora aproximadamente; (d) el grupo instruccional está formado por uno o más alumnos (no más de cinco) y un profesor que actúa como mediador del aprendizaje; (e) una vez definido el tema o problema de estudio, el tutor asigna tareas específicas al estudiante, las cuáles son ejecutadas por éste y sus resultados son evaluados y retroinformados por aquél posteriormente; (f) el alumno aprende por medio de tres diferentes actividades: primero, al hacer su trabajo él solo; segundo, en la interacción con el tutor, al observar y reflexionar sobre los errores cometidos y defender los puntos de vista que considera 
acertados; $y$ tercero, al revisar críticamente el trabajo completo, corregirlo y compararlo con las versiones anteriores. (págs. 50$51)$.

Ahora bien, es importante destacar que estos elementos representan las bases de lo que hasta ahora se conoce como tutoría en Venezuela y que se encuentra tipificada (como se refirió explícitamente en párrafos anteriores) dentro de la normativa planteada por el CNU (2001c): función que sigue siendo materia de investigación y, por consiguiente, de transformación para que se adapte a la satisfacción de las necesidades, requerimientos, demandas y exigencias reales, especialmente en el contexto educativo. No obstante, el sistema tutorial no es de uso exclusivo en el sistema universitario; pues en otros países, el mismo es utilizado en otros niveles y modalidades del sistema educativo (como primaria y media); en Venezuela predomina la práctica tutorial en el sentido ya descrito y sobre el que se centrará el abordaje referencial de esta publicación.

Se precisa entonces que en Venezuela el énfasis marcado de la tutoría es en el contexto universitario, muy especialmente en el subsistema de educación universitaria a nivel de postgrado; pero la misma es concebida más como una función a desempeñar, pues se le utiliza esencialmente, según se infiere de CNU (2001d): como táctica o estrategia obligatoria en todas las Universidades públicas y privadas para que el estudiante aprenda a investigar por medio de la elaboración de su Trabajo de Grado.

En el caso de algunas Universidades autónomas y experimentales del país, en cuyos Reglamentos de Postgrado se tipifica que durante el diseño y desarrollo de los Trabajos de Grado, los estudiantes deben contar con la asistencia de un Tutor. Esto es un aspecto importante porque guarda relación con algunas características del sistema tutorial inglés enfocándose en una atención casi personalizada que permita el desarrollo de una relación tutorestudiante para promover la comprensión y abordaje de la investigación no 
solo como un requisito de grado, sino como una vía expedita y lo menos traumática posible para solucionar los problemas en el entorno profesional y social; en fin, el docente universitario asignado para tal fin, cumple una función específica de acompañamiento al estudiante para la elaboración de su producto académico, el cual figura como un requisito para la obtención del título correspondiente.

Ahora bien, esto implica reflexionar sobre algunos requerimientos y características de quienes cumplen la función tutorial en las universidades venezolanas, por cuanto el propósito nomotético que se aspira lograr con su actividad está fundamentado en el acompañamiento para la elaboración de un Trabajo de Grado; no obstante, más allá de eso, es importante establecer una relación entre el Tutor y el tutorado que permita contribuir al desarrollo de un proceso investigativo que trascienda el cumplimiento de un requisito de grado y se convierta en la adquisición de habilidades profesionales tendientes a la investigación.

Uno de los requisitos establecidos para un tutor en el nivel de postgrado, es que como mínimo posea el título correspondiente al nivel para el cual aspira ser Tutor; exigencia que no es suficiente por cuanto, tener un título de postgrado no es una garantía para desempeñar eficazmente funciones y actividades tutoriales de calidad. Este primer requisito referido al título de posgrado hace énfasis en un producto mas no necesariamente garantiza la experiencia reflexiva y procesual inherente a la función tutorial, por lo cual el desarrollo de competencias tutoriales va más allá de ese aspecto y comprende un conjunto de experiencias, estrategias y destrezas necesarias para ese desempeño.

Sin que ésta sea el único requerimiento, surgen para el Tutor un conjunto de competencias producto de su capacitación, práctica y experiencia de investigación en el ámbito de incumbencia del Trabajo de Grado; esto es fundamental puesto que la producción del hacer investigativo exige 
conocimiento experiencia específicas al área de cada posgrado. Por tanto, la acción investigativa se presenta como un requisito imprescindible para el profesional que ejerza dicha función. Este aspecto específico es un elemento de relevante preocupación dentro de la gestión administrativa de los gerentes universitarios ya que ubicar personal académico idóneo con este perfil trasciende a únicamente cumplir con el primer requisito (tener el título necesario para el nivel de postgrado en el que se espera trabajar); pues ser investigador requiere de una labor continuada y sistemática, es una condición adquirida con la práctica, es una actitud ante el entorno, una forma de vida que filtra su cosmovisión con base en esa dinámica: investigar. De allí que ser investigador, per se, es uno de los requisitos más difíciles de encontrar en los Tutores, pero uno muy necesario para el desarrollo de óptimas competencias tutoriales.

Esta serie de criterios para ser Tutor, representan un elemento normativo para el desarrollo de las actividades de postgrado. Pero una visión profunda sobre el tema apunta hacia un conjunto de competencias de tipo profesional y personal como elementos esenciales para un óptimo desempeño como Tutor. Al respecto, Díaz (2008): plantea que el Tutor constituye un factor determinante para el éxito o fracaso en la elaboración del Trabajo de Grado porque es el encargado de brindar al tutorado las orientaciones necesarias para desarrollar el proceso de investigación; de esto se deduce que la tutoría va más allá de un proceso meramente académico y requiere de un conjunto de competencias que permitan alcanzar el propósito estratégico para el cual se le contrata, que es coadyuvar en la elaboración del Trabajo de Grado.

De igual manera resalta lo planteado por Ruiz (2006c), cuando señala que:

La mayoría de las instituciones que desarrollan programas de postgrado enfrentan un problema... Se trata de la insuficiencia 
de tutores con experiencia, tanto en el ejercicio de la investigación, como en la supervisión y asesoramiento de trabajos de investigación realizados por terceros. En el caso de los programas de postgrado en Educación, ésta es una situación muy generalizada en todo el país, lo cual tiene dos consecuencias... a). la posibilidad de que se incremente el fenómeno TMT y/o b). que se lleguen a graduar estudiantes con una formación académica de muy dudosa calidad, particularmente en lo que respecta al desarrollo de las competencias como investigador (págs. 39-40).

En este orden de ideas, es fundamental considerar que no se puede dar lo que no se tiene; es decir, un Tutor no puede desarrollar competencias investigativas en los estudiantes, si él mismo no las posee. Además, que dichas competencias investigativas vienen a ser la base para desarrollar competencias tutoriales, las cuales permite el cumplimiento de uno de los requisitos básicos establecidos por el CNU (1993): para autorizar el funcionamiento de los Programas de Postgrado como es "...disponer de personal de planta suficiente y con la formación adecuada para asegurar...la dirección de trabajos de grado o tesis doctorales..." (Numeral 5).

Esta formación implica que el Tutor debe contar con un conjunto de competencias tutoriales que le permitan satisfacer las demandas planteadas, especialmente una adecuada orientación al estudiante o tutorado en relación con la elaboración de su Trabajo de Grado. Dichas competencias, esencialmente, se forjan en la orientación que se les brinda a los estudiantes para la producción de los textos académicos requeridos en este proceso de formación como investigador novel (artículos científicos, Proyectos de Trabajo de Grado y Tesis Doctoral). Como refieren Cruz, Díaz y Abreu (2010):

El trabajo tutorial en los estudios de posgrado resulta imprescindible para la formación de futuros investigadores, pues posee un alto potencial para revitalizar el saber, integrar redes de colaboración y posicionar nuevos líderes en la generación, innovación y transferencia del conocimiento. Sin embargo, la tarea de los tutores no resulta sencilla, pues se carece de 
instrumentos que guíen su desempeño; es por ello que en ocasiones rigen su actuar basados en sus propias creencias y experiencias previas, sin una reflexión continua de su quehacer como formadores (pág. 83).

Tal aseveración representa un aporte importante pues revela como las vivencias, creencias y experiencias de la cotidianidad brindan un aporte fáctico a la noción de función tutorial. No obstante, por sí solas, sin la sistematización, reflexión, análisis y comprensión generadas desde un proceso de investigación se convierten en procesos aislados que pudieran o no aportar con veracidad y asertividad a la dinámica de producción de textos académicos en el nivel de postgrado.

Ahora bien, sabiendo la importancia del Tutor para la realización de los Trabajos de Grado en los Programas de Postgrado Venezolanos, se hace necesaria su conceptualización. Para Ruiz (1996): el Tutor de Trabajos de Grado es "... un docente - investigador que, en su carácter de experto, tiene la responsabilidad de dirigir, de manera competente, la actividad académica del estudiante asociado con todo el proceso de elaboración de su trabajo o tesis de grado" (pág. 56). De allí se puede colegir cómo la función tutorial en el nivel de posgrado, además de ajustarse a un contexto científico y académico particular como parte de la gestión del conocimiento, requiere responder a las demandas y exigencias sociales del momento, promoviendo alternativas para reencaminar el transitar social en el contexto del progreso y desarrollo.

Aunado a ello, se espera que el Tutor contribuya primordialmente en el proceso de formación de investigadores nóveles; esto requiere un conjunto de elementos e intercambios propios de la vida académica de cualquier Programa de Postgrado, en los que procesos como la alfabetización académica juegan un papel fundamental de apoyo para el cumplimiento de la función tutorial, así como el desarrollo de competencias en esta área. 
En este orden de ideas, es importante precisar una tipología de los Tutores. Por ejemplo, Terán (2012b), refiere que:

... se pueden identificar tres tipos de tutores: a). experto, b). consolidado y c). novel. a). El tutor experto, se considera al profesional con un perfil de desempeño en las categorías que definen la competencia tutorial, esto es: profundo conocimiento en teorías y epistemología de la investigación, experiencia como investigador y amplio conocimiento en metodología y estrategias de asesoría académica... b). El perfil del tutor consolidado, es aquel profesional que ha adquirido una cierta experiencia en el desempeño como tutor de trabajos de grado o tesis, conocimiento limitado de epistemología de la investigación, y poca experiencia como investigador. c). Finalmente, el tutor novel es aquel profesional que tiene poca o ninguna experiencia como investigador y limitada experiencia como asesor de trabajos de investigación y un dominio moderado en epistemología y teorías de la investigación (pág. $60)$.

Dicha caracterización ofrece una visión global acerca de los aspectos más resaltantes requeridos por un Tutor. Asimismo, esta expresa los tipos de Tutores con los que se cuenta para apoyar la administración curricular y elaboración de los Trabajos de Grado por parte en las Universidades, dando un papel preponderante al ejercicio de la investigación (y todos los procesos que le acompañan), como aspecto fundamental para desempeñarse adecuadamente como Tutor.

\subsection{Instrumentación de la Competencia Tutorial: perspectivas encontradas}

Sobre este punto es imprescindible consultar el aporte realizado por Valarino (1997): en la operacionalización de la competencia tutorial, cuando declara de manera sucinta las dimensiones de la tutoría: a). funciones del rol (la cual atañe al conocimiento y puesta en práctica de las responsabilidades del tutor en relación con el cumplimiento de las asesorías y demás roles 
demandados en el ejercicio de su labor, lo que en esencia se traduce en

modelar al estudiante y brindarle apoyo como investigador activo); b). competencia (ámbito complejo y dinámico que se refiere a la experiencia y conocimientos del tutor en el proceso de investigación, evidenciando así un conjunto de destrezas no solo en lo epistémico, metodológico u ontológico sino también en la comunicación y manejo adecuado de las relaciones interpersonales, así como la planificación y administración de información sobre el proceso de investigación); c). condiciones emocionales (ámbito complejo que requiere la apertura afectiva e interés a nuevas experiencias, así como la tolerancia a la fase de iniciación en la que posiblemente se encuentra el estudiante). A modo de confirmación, Terán (2012c), manifiesta que:

La tutoría desempeña un papel preponderante en el desarrollo de la investigación del trabajo de grado o tesis, varía según la competencia y desempeño del tutor; la misma que se operacionaliza con base a tres categorías: a). conocimiento teórico-epistemológico; b). experiencia como investigador; y c). experiencia en asesoría y acompañamiento académico... (pág. 59).

Ahora bien, estos tres elementos son muy similares a los planteados por Ruiz (2006d), quien refiere que la competencia tutorial puede ser instrumentalizada de la forma siguiente:

a). Amplio y profundo conocimiento de la temática objeto del Trabajo de Grado, lo cual cuenta con tres dimensiones a saber: a.1.). formación académica (dependiente del grado científico que se tenga); a.2). experiencia profesional (años de ejercicio); a.3). experiencia como Tutor (número de Trabajos de Grado asesorados).

b). Experiencia como investigador en el área temática, con dos dimensiones que la conforman: b.1). conocimiento declarativo de aspectos teóricos como paradigma, ontología, metodología o teorías específicas; b.2). conocimiento procedimental, relacionado con saber cómo plantear el 
problema, elaborar el marco teórico, describir el método, presentar los resultados, elaborar la discusión y presentar las conclusiones, elaborar el informe.

c). Tener experiencia como Asesor Académico, lo cual se estructura en tres dimensiones: c.1). método de asesoramiento que implica el motivo de la solicitud de ayuda, identificación del problema, análisis de factibilidad, instrumentación, seguimiento; c.2). manejo de la comunicación y control de las emociones; c.3). uso o no de la mediación cognitiva.

Estas dimensiones vienen a marcar una pauta en el establecimiento de una caracterización del desempeño de la figura del tutor, la cual sugiere un conjunto de aspectos importantes para la reflexión; en el caso de Balbi (2011b), la competencia tutorial:

...Se evidencia a través de un conjunto de acciones técnicasacadémicas, de comunicación científica y profesionales en las que intervienen los factores cognitivos y afectivos del tutor y que conjuntamente con el estudiante crea las condiciones para que pueda alcanzar la meta de culminar el trabajo de investigación al mismo tiempo que se forma o entrena en los procedimientos metodológicos. Esto supone que las acciones del tutor son estratégicas y dirigidas a facilitar el proceso, ayudándole a anticipar obstáculos y a tomar decisiones oportunas que lo conduzcan a alcanzar la meta (pág. 24).

La cita precedente plantea una función tutorial que abarca el ejercicio en variadas áreas que van desde lo académico (técnico, profesional, cognitivo) hasta lo relacional (emocional, comunicacional); en estos ámbitos el trabajo del tutor necesariamente debe ser consciente e intencional con el fin de mediar el proceso o acción tutorial. Ha de resaltarse este aspecto pues tradicionalmente destaca el proceso de autoaprendizaje del estudiante por encima de la función tutorial, siendo que en la realidad ambos se complementan, pero el primero depende en gran manera de la claridad y 
asertividad en las acciones del tutor.

El Tutor requiere fomentar y consolidar en el estudiante una disciplina, acuciosidad y profundización en el trabajo intelectual, de manera tal que éste tome conciencia de la complejidad del proceso y asuma una actitud dinámica, crítica, creativa y analítica que le permita capacitarse en el hecho investigativo, valorar y potencializar sus capacidades y habilidades como profesional y sujeto social activamente responsable. Desde esta representación, se asume la concepción de competencia tutorial planteada por Ruiz (2006e), quien declara que es:

...La expresión integral de factores específicos de las dimensiones cognitiva (conocimientos y habilidades) y afectiva (actitudes, valores y confianza en sí mismo) que exhibe un docente-investigador, a través de su desempeño profesional, durante el proceso de dirigir, asesorar y supervisar a un estudiante en la elaboración de su trabajo o tesis de grado...en consecuencia, la competencia tutorial, en tanto que desempeño, se traduce en un conjunto de acciones técnicoacadémicas, comunicacionales, científicas y profesionales, mediadas por factores cognitivos y afectivos del tutor, que pone en práctica, previo acuerdo con el estudiante, con el propósito deliberado de crear las condiciones apropiadas para que el sujeto logre la meta de hacer una tesis con calidad y pertinencia social, al mismo tiempo que se entrena en los métodos, técnicas y procedimientos de investigación (pág. 70).

De acuerdo con lo antes expuesto, en la competencia tutorial se conjugan tanto el conocimiento del área como la experiencia en la investigación y como tutor. Aunado a ello, Ruiz considera igualmente esencial para el óptimo desempeño de la competencia tutorial, considerar los rasgos característicos del tesista, como son su perfil académico, sus fortalezas, debilidades y el contexto familiar y laboral. La visión expuesta por este autor logra develar las diversas dimensiones de una actuación signada por la dialéctica del saber y el hacer, así como de lo individual y lo social. 


\section{Consideraciones Finales}

En Venezuela, la función tutorial adquiere su expresión como competencia específicamente universitaria. El creciente desarrollo del sector de estudios de postgrado en Venezuela ha llevado a la indagación acerca de cuáles son los factores involucrados en la exitosa culminación de dichos estudios. Tradicionalmente la función tutorial en el ámbito universitario ha sido considerada desde una perspectiva unilateral, esto es, una visión de la competencia tutorial sólo vista desde el lado del docente. De ahí la importancia de establecer un panorama más completo de la función tutorial al desarrollar una perspectiva bidireccional en la que se incluye no sólo la competencia y el saber del tutor sino su interrelación con el tesista.

Las múltiples dimensiones involucradas en la competencia tutorial revelan la complejidad de una función medular en la formación de investigadores. Esta realidad en constante construcción en los postgrados venezolanos comporta un camino en sí misma ya que exige de una constante revisión, reflexión y presentación de propuestas en lo relacionado con la concepción, características y cualidades de un tutor competente y efectivo, así como las interacciones en la tutoría en el referido nivel universitario. De allí que la presente contribución representa un acercamiento pertinente en esta área no solo para los Programas de Postgrado en Venezuela, sino en todo el contexto universitario internacional.

\section{Referencias}

Balbi, A. (2011a,b). Diseño de un Programa de Formación de Tutores. Kaleidoscopio, 08(16), 21-30. ISSN: 1690-6054. Universidad Nacional Puerto Ordaz, Venezuela: Experimental de Guayana. Recuperado de: http://kaleidoscopio.uneg.edu.ve/numeros/k16/k16 art02.pdf

Bello, F. (2003). La Política tutorial y el crecimiento de los Estudios de Postgrado en Venezuela (Análisis y Propuestas). Revista Ciencias 
de la Educación, 2(22), 59-78. ISSN: 1316-5917. Recuperado de:

http://servicio.bc.uc.edu.ve/educacion/revista/a3n22/22-4.pdf

Benedito, V., Ferrer, V., \& Ferreres, V. (1995). La formación universitaria a

debate. Barcelona: Publicaciones de la Universidad de Barcelona.

Consejo Consultivo Nacional de Postgrado (2018). Estadísticas. Ministerio del Poder popular para Educación Universitaria, Ciencia y Tecnología.

Caracas, Venezuela: Consejo Nacional de Universidades. Recuperado de: http://www.ccnpg.gob.ve/estadisticas/

Consejo Nacional de Universidades (1993). Resolución referida a la Política Nacional de Estudios de Postgrado. Gaceta Oficial № 35.210 de fecha 27 de marzo. Caracas, Venezuela: Consejo Nacional de Universidades.

Consejo Nacional de Universidades (2001a,b,c,d). Normativa General de los Estudios de Postgrado para las Universidades e Instituciones debidamente autorizadas por el Consejo Nacional de Universidades. Gaceta Oficial № 37.328 de fecha 20 de noviembre. Caracas, Venezuela: Consejo Nacional de Universidades.

Cruz, G., Díaz, A., \& Abreu, L. (2010). La Labor Tutorial en los estudios de postgrado. Rúbricas para guiar su desempeño y evaluación. Perfiles Educativos, XXXII(130), 83-102, ISSN: 0185-2698. Distrito Federal, México: Instituto de Investigaciones sobre la Universidad y la Educación. Recuperado de:

http://www.redalyc.org/articulo.oa?id=13214992006

Díaz, S. (2008). Perfil ideal de Competencias del Tutor de Trabajos a nivel de Postgrado de la Facultad de Ciencias de la Educación de la Universidad de Carabobo. ARJÉ Revista de Postgrado FACE-UC, 1(2), 154-182. ISSN digital: 2443-4442, ISSN impreso: 1856-9153. Recuperado de: http://www.arje.bc.uc.edu.ve/arj02/art07.pdf

López, C. (2016). Didáctica Conversora del Conocimiento: Construcción 
de un Modelo integrativo en Educación Universitaria. Observador del Conocimiento 3(4), 39-44, ISSN: 2343-6212. Recuperado de: https://issuu.com/oncti/docs/ocv3n4

Rondón, A. (2013). El tutor y los procesos de tutoría en las universidades venezolanas. España: Editorial Académica Española, ISBN-13: 978-3659-07392-2, ISBN-10: 365907392X, EAN: 9783659073922, págs. 56. Recuperado de: http://www.eae-publishing.com/

Ruiz, C. (1996). La Competencia Tutorial. Un análisis teórico-conceptual. Planiuc, 14(22), 93-118, ISSN: 0253-6617.

Ruiz, C. (2006a,b,c,d,e). Cómo llegar a ser un tutor competente. Caracas, Venezuela: Aula XXI, Santillana/UPEL.

Terán, G. (2012a,b,c). Los Trabajos de Grado y niveles de productividad en los Postgrados: Aproximación Teórica de Seguimiento Tutorial. Eidos, (5), 59-67, ISSN: 1390-5007. Quito, Ecuador: Universidad Tecnológica Equinoccial. Recuperado de:

https://doi.org/10.29019/eidos.v0i5.96

Valarino, E. (1997). Tesis a Tiempo. Caracas, Venezuela: Equinoccio, Ediciones de la Universidad Simón Bolívar, Impresión: Editorial Melvin, ISBN: 980-237-141-6, págs. 365. Recuperado de:

https://books.google.co.ve/books?id=W vA02P3obgC\&printsec=frontco $\underline{\text { ver\# } \mathrm{v}=\text { onepage } \& \mathrm{q} \& \mathrm{f}=\text { false }}$ 


\section{Carmen Consuelo López De Solórzano \\ e-mail: clopez@impm.upel.edu.ve}

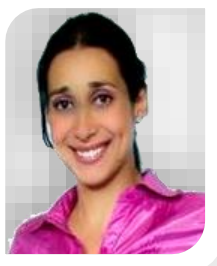

Nacida en Venezuela, Profesora en Educación Preescolar (UPEL, 1999), Magister Scientiarum en Educación Abierta y a Distancia (UNA, 2010) y Doctora en Ciencias de la Educación (UFT, 2014). Docente Asociado a Dedicación Exclusiva de la UPEL, adscrita a la Extensión Académica Barinas del Instituto de Mejoramiento Profesional del Magisterio. Entre sus temáticas de investigación destacan: Didáctica Universitaria, Competencias Tutoriales, Cultura Investigativa y Necesidades de Capacitación Docente. Actualmente se desempeña como Coordinadora Local de los Programas de Investigación y Postgrado y del Núcleo de Investigación Educativa Barinas. Publica artículos científicos en revistas científicas y participa como Conferencista, Ponente y Tallerista en actividades académicas. 


\section{Mariela Sofía Pérez Rodríguez \\ e-mail: marielasofi@gmail.com}

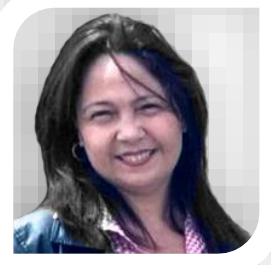

Nacida en Venezuela, Licenciada en Letras, mención Lenguas y Literaturas Clásicas ULA (1996). Magister en Lingüística de la UPEL-IPB (2002). Docente Agregado Dedicación Exclusiva de la UPEL, adscrita al Dpto. de Castellano y Literatura donde ha administrado cursos relacionados con historia de la lengua, la gramática, la comprensión y producción textual. Investigadora adscrita a las líneas Análisis del Discurso y Literatura latinoamericana del Núcleo de Investigación Lingüística Literaria Trino Borges. Coordinadora de la Maestría en Lingüística del IPB. Ha publicado artículos en revistas especializadas. Actualmente cursa el Doctorado en Cultura Latinoamericana y Caribeña de la UPEL-IPB.

El contenido de este manuscrito se difunde bajo una Licencia de Creative Commons Reconocimiento- 